\title{
The Simpson grading revisited: aggressive surgery and its place in modern meningioma management
}

\author{
Konstantinos Gousias, MD, DSc, ${ }^{1,2}$ Johannes Schramm, MD, ${ }^{1}$ and Matthias Simon, MD1 \\ 1Department of Neurosurgery, University Hospital of Bonn; and 2Department of Neurosurgery, University Hospital of Bochum \\ Bergmannsheil, Bochum, Germany
}

\begin{abstract}
OBJECTIVE Recent advances in radiotherapy and neuroimaging have called into question the traditional role of aggressive resections in patients with meningiomas. In the present study the authors reviewed their institutional experience with a policy based on maximal safe resections for meningiomas, and they analyzed the impact of the degree of resection on functional outcome and progression-free survival (PFS).

METHODS The authors retrospectively analyzed 901 consecutive patients with primary meningiomas (716 WHO Grade I, 174 Grade II, and 11 Grade III) who underwent resections at the University Hospital of Bonn between 1996 and 2008. Clinical and treatment parameters as well as tumor characteristics were analyzed using standard statistical methods.

RESULTS The median follow-up was 62 months. PFS rates at 5 and 10 years were $92.6 \%$ and $86.0 \%$, respectively. Younger age, higher preoperative Karnofsky Performance Scale (KPS) score, and convexity tumor location, but not the degree of resection, were identified as independent predictors of a good functional outcome (defined as KPS Score 90100). Independent predictors of PFS were degree of resection (Simpson Grade I vs II vs III vs IV), MIB-1 index (<5\% vs $5 \%-10 \%$ vs $>10 \%$ ), histological grade (WHO I vs II vs III), tumor size ( $\leq 6 \mathrm{vs}>6 \mathrm{~cm}$ ), tumor multiplicity, and location. A Simpson Grade II rather than Grade I resection more than doubled the risk of recurrence at 10 years in the overall series (18.8\% vs $8.5 \%$ ). The impact of aggressive resections was much stronger in higher grade meningiomas.

CONCLUSIONS A policy of maximal safe resections for meningiomas prolongs PFS and is not associated with increased morbidity. http://thejns.org/doi/abs/10.3171/2015.9.JNS15754
\end{abstract}

KEY WORDS Simpson grade of resection; recurrence; skull base meningioma; non-skull base meningioma; oncology

$\mathrm{M}$ ENINGIOMAS account for about one-third of primary brain tumors. ${ }^{11}$ Surgery has long been the treatment of choice (and also more or less the only therapeutic option) for most patients. In 1957 Simpson published his classification of the extent of resection and suggested more aggressive operative strategies as the key to minimizing the risk of recurrence. ${ }^{18}$ Ever since, many studies have confirmed this basic relationship between the extent of resection and meningioma recurrence, which underlies the belief that meningioma surgery should aim at a radical resection of the tumor, its dural attachments, and any other structure infiltrated by the meningioma..$^{5,10,13}$ Not all meningiomas are benign tumors.

Neuropathologists assign between $10 \%$ and $20 \%$ of tumors to WHO Grades II and III. ${ }^{8}$ There is a sizable body of data demonstrating a close correlation between WHO grade and tumor recurrence. Nevertheless, even some of the higher grade tumors seem to be curable through resection.

More recently, many neurosurgeons have started to routinely use less aggressive resection strategies. Radiosurgery and stereotactic radiotherapy can often be successfully used to control difficult-to-resect tumor remnants. ${ }^{3,6}$ The widespread availability of high-quality neuroimaging allows for safe follow-up of residual tumor. Many believe that more conservative surgery in combination with radiotherapy and/or serial imaging follow-up will result in good tumor control and better clinical outcomes than the more traditional aggressive surgical approach. ${ }^{2}$

Even the clinical relevance of Simpson's classification of the extent of resection has been questioned. Sughrue et al. found no statistically significant difference in recurrence-free survival between patients following a Simpson Grade I, II, III, or IV resection for benign meningiomas. ${ }^{19}$ 
Taken at face value, these data suggest that something like a simple resection of the tumor mass or even mere tumor debulking should be regarded as the surgical treatment of choice for benign meningiomas. The authors speculated that the discrepancy between their findings and other series may reflect the more recent improvements in surgical and imaging techniques, which have likely resulted in very different tumors assigned to the various Simpson grades compared with earlier decades.

Treatment for meningiomas at our institution has been based for many years on the tenets of the degree of resection/risk of recurrence relationship. We have consistently followed a policy of maximal safe resection and have opted for aggressive surgery whenever such a strategy was felt to reduce the risk of recurrence without unduly increasing the risk of neurological sequelae or other complications. On the other hand, we have also been keenly aware of the limitations of aggressive meningioma surgery. ${ }^{20}$ For the present paper, we have analyzed our experience with this policy in a series of 901 patients. Our results show that the degree of resection (as measured by the Simpson grade) is a powerful predictor of tumor recurrence even in a contemporary patient series and therefore still conveys useful clinical information. Our data also seem to suggest that aggressive surgery (within the limits of the policy detailed above) is not associated with significantly increased rates of neurological and other complications.

\section{Methods}

\section{Patients and Clinical Data}

For the present study we identified all patients who were 18 years or older in the departmental tumor (tissue) bank with a histologically confirmed meningioma who underwent surgery at our institution between 1996 and 2008. We excluded patients presenting with tumor recurrence who underwent their primary surgery at an outside institution and all patients for whom the degree of resection (Simpson grade) at their initial operation was not documented. Pertinent clinical and treatment data (age, sex, preoperative and postoperative Karnofsky Performance Scale [KPS] scores, tumor size and location, tumor multiplicity, degree of resection, and radiotherapy/radiosurgery), and follow-up information were obtained through a retrospective chart review. Specifically, the degree of resection was assessed by the first and the senior authors, who reviewed all operative notes and radiological reports. The study was approved by the University of Bonn Medical Center institutional review board for human research.

According to routine, patients were followed through our outpatient department and underwent yearly MRI for at least 3 years (and at longer intervals thereafter). Intervals between MRI studies were at least 4-6 months during the first 1-2 years following surgery for a WHO Grade II or III meningioma. Additional follow-up information was obtained through structured questionnaires sent to the patients and/or their family physicians. Tumor recurrence or progression was assessed by the treating neurosurgeon and neuroradiologist and confirmed by the interdisciplinary tumor board, and was defined as tumor (re)growth (of the index meningioma in cases with multiple meningio- mas) shown on follow-up MRI with or without a clinical correlate.

\section{Neuropathology}

All histopathological diagnoses were made at the Institute for Neuropathology of the University of Bonn Medical Center/German Brain Tumor Reference. Tumor grading followed the WHO classification as valid at the time of surgery. In an attempt to account for the impact of revisions of the classification, patients who were treated before and after 2000 were also analyzed separately (see below). Since the only major change introduced by the WHO 2007 revision appears to be the inclusion of brain invasion among the criteria sufficient to diagnose atypia ${ }^{8}$ cases treated between 2000 and 2008 with brain invasion but lacking other criteria of anaplasia were retrospectively assigned to WHO Grade II, and all surgeries performed after 2000 were analyzed together. MIB-1/Ki 67 immunohistochemistry was performed as previously described., ${ }^{4,15,16}$

\section{Statistical Analysis}

All statistical analyses were performed using commercially available software (SPSS version 21, IBM Deutschland). Survival end points (progression-free survival [PFS] and overall survival [OS]) were analyzed with KaplanMeier estimates using the log-rank test for comparisons. PFS was also studied using univariate and multivariate Cox proportional hazards modeling. Patient outcome was analyzed using univariate and multivariate regression analysis. Multivariate testing routinely included those variables that were statistically significantly ( $p<0.05,2$-tailed) associated with the end point in question.

\section{Results}

\section{Patient Demographics and Tumor Characteristics}

This study comprised 901 patients; $70.8 \%$ of patients were female. The median age at the time of the patients' first surgery was 60.8 years (mean $59.9 \pm 12.8$ years, range 20.2-92.1 years). Fifty-eight patients (6.4\%) were diagnosed either initially or during follow-up with more than one meningioma, including 43 patients $(4.8 \%)$ with 2 tumors, 10 (1.1\%) with 3 tumors, and 1 patient with 4 tumors. Four patients $(0.4 \%)$ harbored more than 5 distinct meningiomas. We studied only 1 tumor per patient. Overall, $36.2 \%$ of (index) tumors measured smaller than $3 \mathrm{~cm}$ and $10.1 \%$ larger than $6 \mathrm{~cm}$. Information on tumor location can be found in Table 1 .

The series includes 716 WHO Grade I (79.5\%), 174 WHO Grade II (19.3\%), and 11 WHO Grade III meningiomas (1.2\%). A total of 696 (77.2\%) of histopathological diagnoses were made using the WHO 2000/2007 classifications (see Methods). MIB-1 indices were available for $741(82.2 \%)$ of cases. Seventy-one tumors $(9.6 \%)$ had an MIB-1 index < 2\%, $503(67.9 \%)$ an MIB-1 index of 2\% to $<5 \%, 136(18.4 \%)$ an MIB-1 index of $5 \%-10 \%$, and in 31 tumors (4.2\%) the MIB-1 index was $>10 \%$.

\section{Tumor Treatment and Functional Outcomes}

A Simpson Grade I resection was performed in 572 (63.5\%) of our cases, a Grade II resection in 199 (22.1\%), 
TABLE 1. Location of 901 meningiomas

\begin{tabular}{lc}
\hline \multicolumn{1}{c}{ Location } & No. of Meningiomas $(\%)$ \\
\hline Supratentorial convexity & $214(23.8)$ \\
\hline Infratentorial convexity & $12(1.3)$ \\
\hline Parasagittal & $137(15.2)$ \\
\hline Falx & $84(9.3)$ \\
\hline Tentorium \& falcotentorial & $42(4.7)$ \\
\hline Ventricles & $13(1.4)$ \\
\hline Olfactory groove & $62(6.9)$ \\
\hline Suprasellar & $54(6.0)$ \\
\hline Other frontobasal & $26(2.9)$ \\
\hline Middle sphenoid wing & $49(5.4)$ \\
\hline Lateral sphenoid wing & $45(5.0)$ \\
\hline Temporobasal/cavernous sinus & $22(2.4)$ \\
\hline Sphenoorbital & $18(2.0)$ \\
\hline Petrous bone & $48(5.3)$ \\
\hline Clivus \& petroclival & $19(2.1)$ \\
\hline Foramen magnum & $7(0.8)$ \\
\hline Spinal & $46(5.1)$ \\
\hline Other & $3(0.3)$ \\
\hline Total & $901(100.0)$ \\
\hline
\end{tabular}

and Grade III and IV resections in 93 (10.3\%), and 37 (4.1\%) patients, respectively.

During the study period, radiotherapy was routinely prescribed in cases of WHO Grade III anaplastic meningiomas. Radiosurgery or radiotherapy was also offered to patients with meningiomas WHO Grades I/II and residual tumor following a Simpson Grade IV resection (either as part of their primary treatment or at the time of tumor progression). Overall, 31 patients (3.5\%) had radiation therapy as part of their primary treatment. Thirteen patients had conventional fractionated radiotherapy and 18 patients underwent Gamma Knife surgery or stereotactic radiosurgery (including one patient who had upfront radiosurgery). Eight patients had adjuvant radiotherapy for a Grade III tumor. Five patients with atypical meningiomas had radiotherapy/radiosurgery following a Simpson Grade III resection $(n=3)$ or for a tumor initially (i.e., using pre-WHO 2000 criteria; $n=2$ ) assigned to WHO Grade III. Fifteen patients with WHO Grade I tumors had radiation therapy for residual tumor (i.e., following a Simpson Grade III/ IV resection). Three patients underwent radiation therapy at outside institutions because of postoperative imaging findings suggesting residual tumor despite the surgical impression of a Simpson Grade I/II resection.

The median postoperative KPS score was 80 (mean $80.5 \pm 17.6$ [preoperative KPS score: median 90, mean $83.4 \pm 13.5]$ ). The preoperative KPS score proved to be the most powerful predictor of the patients' postoperative functional outcome (preoperative KPS Score 90-100 vs postoperative KPS Score 90-100; HR 35.814 [95\% CI 24.200-53.003], $\mathrm{p}<0.001)$. Of note, the Simpson grade varied significantly with the postoperative KPS score. Interestingly, more aggressive resections (Simpson Grade I vs II vs III vs IV) were associated with a better rather than lower postoperative performance score. As a corollary, new postoperative neurological deficits or worsening of preexisting ones also correlated inversely with the Simpson grade (both $\mathrm{p}<0.001$ ). The overall rate of new or worsened focal neurological deficits was $9.3 \%$. Patients with tumors of the cerebral convexity had significantly better outcomes, and those with petroclival and spinal meningiomas and meningiomas of the foramen magnum had relatively adverse outcomes. Additional significant outcome predictors in the univariate analysis included patient age, tumor size, and the degree of resection (Table 2).

A multivariate regression with all parameters correlating significantly with the postoperative KPS score in the univariate analysis revealed only age, the preoperative KPS score, and a convexity or petroclival tumor location as independent predictors of the patients' functional outcomes. Again, the preoperative KPS score was identified as the most important prognostic parameter (Table 3). The degree of resection did not independently impact the patients' functional outcomes.

\section{Follow-Up and Tumor Recurrence}

The median follow-up was 62 months (mean $68.3 \pm$ 48.3 months) for the overall series; 284 (31.5\%) of the patients were followed until death or for at least 10 years. Estimated PFS at 5 and 10 years was $92.6 \%$ and $86.0 \%$, respectively. Only one recurrent tumor was diagnosed after longer than 10 years of follow-up. At the time of tumor recurrence or progression, surgery, radiotherapy/radiosurgery, or a combination of both was offered depending on the size, location, and the presumed surgical versus radiotherapy risks, and patient factors such as age and comorbidities. However, if safely possible, surgery was considered the treatment of choice.

Estimated OS (including all causes of death) at 5 and 10 years was $89.6 \%$ and $78.9 \%$, respectively, for the overall series. For comparison, OS at 5 and 10 years for an age- and sex-matched but otherwise unselected German population is $92.6 \%$ and $83.0 \%$, respectively (see www. destatis.de/DE/ZahlenFakten.html); (surgical) meningioma patients have a slightly lower life expectancy compared with the overall population. A total of 125 patients (13.9\%) died during follow-up. This figure includes 7 early $(<4$ weeks) postoperative deaths (an operative mortality of $0.8 \%$ [7 of 901]) and only 12 confirmed tumor-related deaths (or treatment for tumor recurrence; i.e., the majority of deaths seen after meningioma surgery were not directly related to the disease or its treatment). However, OS did vary significantly with the WHO grade of the tumors (Fig. 1 upper). The Simpson grade of resection did not influence OS significantly (for all causes of death) in the overall series (Fig. 1 lower) or in subgroups defined by their histological grade (i.e., WHO Grade I, WHO Grade II, or WHO Grades II/III).

Tumor recurrence rates varied strongly with the histological tumor grade, the MIB-1 labeling index, and the degree of resection (Simpson grade; Table 4, Figs. 2 and 3). PFS rates at 5 years were $95.7 \%, 81.8 \%$, and $46.7 \%$ for WHO Grade I, II, and III meningiomas (Fig. 2 left, $\mathrm{p}<0.001$ ). The 10 -year figures for Grades I and II were $90.4 \%$ and $69.4 \%$. Overall, tumor recurrence was ob- 
TABLE 2. Predictors of a good surgical outcome: univariate analysis

\begin{tabular}{|c|c|c|c|c|c|}
\hline Variable & No. of Patients* & KPS Score 90-100 & $p$ Value & OR & $95 \% \mathrm{Cl}$ \\
\hline Age in yrs & & & $<0.001$ & 0.389 & $0.297-0.510$ \\
\hline$\geq 61$ & 448 & $154(34.4 \%)$ & & & \\
\hline$<61$ & 446 & $256(57.4 \%)$ & & & \\
\hline Preop KPS score & & & $<0.001$ & 35.814 & $24.200-53.003$ \\
\hline $90-100$ & 466 & $369(79.2 \%)$ & & & \\
\hline$\leq 80$ & 427 & $41(9.6 \%)$ & & & \\
\hline Sex & & & 0.115 & 0.791 & $0.591-1.058$ \\
\hline Male & 261 & $109(41.8 \%)$ & & & \\
\hline Female & 633 & $301(47.6 \%)$ & & & \\
\hline \multicolumn{6}{|l|}{ Location } \\
\hline Supratentorial convexity & 211 & $119(56.4 \%)$ & $<0.001$ & 1.742 & $1.276-2.379$ \\
\hline Parasagittal & 137 & $67(48.9 \%)$ & 0.437 & 1.155 & $0.803-1.663$ \\
\hline Falx & 84 & $36(42.9 \%)$ & 0.562 & 0.874 & $0.555-1.376$ \\
\hline Olfactory groove/frontobasal & 88 & $43(48.9 \%)$ & 0.552 & 1.143 & $0.736-1.775$ \\
\hline Suprasellar & 54 & $23(42.6 \%)$ & 0.619 & 0.868 & $0.498-1.515$ \\
\hline Lateral sphenoid & 45 & $19(42.2 \%)$ & 0.615 & 0.856 & $0.467-1.570$ \\
\hline Medial sphenoid & 49 & $16(32.7 \%)$ & 0.059 & 0.555 & $0.301-1.024$ \\
\hline Sphenoorbital & 18 & $6(33.3 \%)$ & 0.287 & 0.584 & $0.217-1.570$ \\
\hline Cavernus sinus/temporobasal & 22 & $10(45.5 \%)$ & 0.969 & 0.983 & $0.420-2.300$ \\
\hline Falcotentorial & 41 & $21(51.2 \%)$ & 0.482 & 1.252 & $0.669-2.345$ \\
\hline Petrous bone & 47 & $22(46.8 \%)$ & 0.893 & 1.041 & $0.578-1.876$ \\
\hline Petroclival & 18 & $2(11.1 \%)$ & 0.010 & 0.143 & $0.033-0.627$ \\
\hline Foramen magnum/spine & 52 & $15(28.8 \%)$ & 0.013 & 0.459 & $0.248-0.849$ \\
\hline All skull base & 346 & $142(41.0 \%)$ & 0.022 & 0.727 & $0.554-0.954$ \\
\hline Multiplicity & & & 0.257 & 0.727 & $0.420-1.261$ \\
\hline Yes & 57 & $22(38.6 \%)$ & & & \\
\hline No & 837 & $388(46.4 \%)$ & & & \\
\hline Tumor size in $\mathrm{cm}$ & & & $<0.001$ & 0.665 & $0.535-0.826$ \\
\hline$<3$ & 315 & $165(52.4 \%)$ & & & \\
\hline $3-6$ & 474 & $207(43.7 \%)$ & & & \\
\hline$>6$ & 87 & $27(31.0 \%)$ & & & \\
\hline WHO grade (all) & & & 0.289 & 0.850 & $0.629-1.148$ \\
\hline I & 711 & $331(46.6 \%)$ & & & \\
\hline ॥ & 172 & $76(44.2 \%)$ & & & \\
\hline III & 11 & $3(27.3 \%)$ & & & \\
\hline WHO 2000/2007 grade & & & 0.376 & 0.856 & $0.606-1.208$ \\
\hline I & 543 & $260(47.9 \%)$ & & & \\
\hline II & 141 & $63(44.7 \%)$ & & & \\
\hline III & 6 & $2(33.3 \%)$ & & & \\
\hline MIB-1 index $(n=741)$ & & & 0.099 & 0.789 & $0.596-1.046$ \\
\hline$<2 \%$ & 71 & $29(40.8 \%)$ & & & \\
\hline 2 to $<5 \%$ & 500 & $243(48.6 \%)$ & & & \\
\hline $5-10 \%$ & 134 & $58(43.3 \%)$ & & & \\
\hline$>10 \%$ & 30 & $10(33.3 \%)$ & & & \\
\hline Simpson grade & & & $<0.001$ & 0.717 & $0.606-0.847$ \\
\hline 1 & 570 & $288(50.5 \%)$ & & & \\
\hline II & 197 & $80(40.6 \%)$ & & & \\
\hline III & 92 & $32(34.8 \%)$ & & & \\
\hline IV & 35 & $10(28.6 \%)$ & & & \\
\hline
\end{tabular}


TABLE 2. Predictors of a good surgical outcome: univariate analysis (continued)

\begin{tabular}{|c|c|c|c|c|c|}
\hline Variable & No. of Patients* & KPS Score 90-100 & p Value & OR & $95 \% \mathrm{Cl}$ \\
\hline Radiosurgery/radiotherapy & & & 0.413 & 0.736 & $0.353-1.535$ \\
\hline Yes & 31 & $12(38.7 \%)$ & & & \\
\hline No & 840 & $388(46.2 \%)$ & & & \\
\hline
\end{tabular}

* Not all data were available for all patients.

served in $4.9 \%$ of WHO Grade I, $18.4 \%$ of atypical with WHO Grade II, and $27.3 \%$ of malignant meningiomas ( $p$ $<0.001$ ). Figures were roughly the same when cases diagnosed before the 2000 revision of the WHO classification were dropped from the analysis. PFS rates at 5 years were $95.0 \%, 94.9 \%, 83.4 \%$, and $60.5 \%$ for tumors with a MIB-1 index $<2 \%, 2 \%$ to $<5 \%, 5 \%-10 \%$, and $>10 \%$, respectively (Fig. 2 right, $\mathrm{p}<0.001$ ). The 5- and 10-year PFS rates for patients undergoing Simpson Grade I, II, III, and IV resections were $96.8 \%$ and $91.8 \%, 91.2 \%$ and $81.2 \%, 76.6 \%$ and $71.8 \%$, and $78.6 \%$ and $65.3 \%$ (Fig. 3A, p < 0.001 ). The corresponding figures after restricting the analysis to 544 patients followed until death or for at least 5 years were roughly the same (Simpson Grade I, 96.8\%/91.8\%; Grade II, 91.9\%/81.8\%; Grade III, 71.8\%/67.3\%; and Grade IV, 78.2\%/65.0\%; $\mathrm{p}<0.001$; Fig. 3B). Interestingly, PFS did not differ between patients with a Simpson Grade I versus Grade II resection for approximately 24 months, i.e., the impact of a Simpson Grade I resection will only be felt during midterm follow-up (Fig. 3A and B).

Tumor size also proved highly predictive of tumor recurrence. Interestingly, increased recurrence rates were only seen when the tumor size exceeded $6 \mathrm{~cm}$ (Table 4). Male patients had worse outcomes than females, possibly reflecting a higher incidence of WHO Grade II/III tumors $(\mathrm{p}<0.001)$. Patients with clival/petroclival tumors had the worst PFS (51.0\% at 10 years) followed by patients with cavernous sinus/temporobasal meningiomas (10-year PFS $70.5 \%$ ). Tumor multiplicity and radiation therapy also correlated with recurrence (Table 4).

Independent predictors of PFS in the multivariate Cox regression analysis included tumor size, WHO grade, the MIB-1 index (<5\% vs $5 \%-10 \%$ vs $>10 \%$ ), tumor location and multiplicity, and the degree of resection (Simpson Grade I vs II vs III/IV; Table 5). The HR for the Simpson grade was close to 2.5 in the multivariate analysis; i.e., increasing the Simpson grade by 1 grade (e.g., performing a Grade II vs a Grade I resection) will generally more

TABLE 3. Predictors of a good surgical outcome: multivariate regression analysis (stepwise backward procedure)

\begin{tabular}{lrrc}
\hline \multicolumn{1}{c}{ Variable } & p Value & \multicolumn{1}{c}{ OR } & $95 \% \mathrm{Cl}$ \\
\hline Age $\geq 61$ yrs & 0.028 & 0.650 & $0.443-0.955$ \\
\hline Preop KPS Score $90-100$ & $<0.001$ & 36.834 & $24.216-56.027$ \\
\hline Location* $^{*}$ & 0.001 & & \\
\hline Supratentorial convexity & 0.001 & 2.292 & $1.435-3.660$ \\
\hline Foramen magnum \& spine & 0.926 & 0.959 & $0.394-2.332$ \\
\hline Petroclival & 0.035 & 0.092 & $0.010-0.847$ \\
\hline
\end{tabular}

* Location: Supratentorial convexity versus foramen magnum \& spine versus petroclival versus all others. than double the risk of recurrence. In the overall series, this translates into a 5\% and $10 \%$ difference in PFS at 5 and 10 years follow-up, respectively, following Simpson Grade I and II resections, respectively. The 5- and 10-year PFS rate differed by 10\%-15\% between Simpson Grade II and III resections (Fig. 3A). One in 5 tumors in this series was assigned to WHO Grade II or III. In absolute numbers the impact of the degree of resection was much stronger in higher grade meningiomas (Fig. 3C and D); in patients with WHO Grade II meningiomas, PFS at 5 years was only $91.1 \%, 78.0 \%$, and $51.2 \%$ following Simpson Grade I, II, and III/IV resections, respectively.
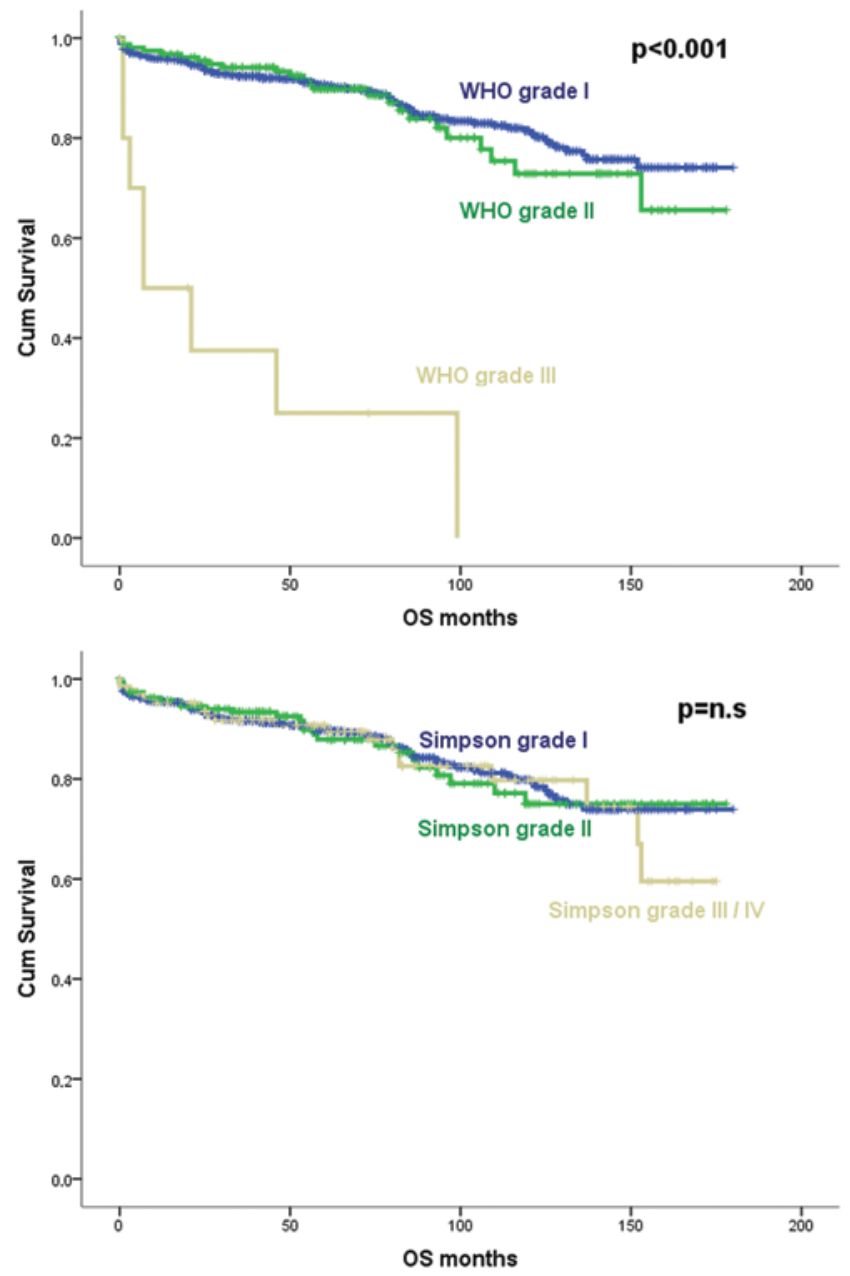

FIG. 1. OS varied with the WHO grade of the tumors (upper) $(p<0.001)$ but not with the extent of resection (Simpson grade) in the overall cohort (lower) ( $p=$ not significant). For illustration purposes, Simpson Grade III and IV resections were combined. Cum = cumulative; n.s = not significant. Figure is available in color online only. 
TABLE 4. Predictors of 10 -year PFS and tumor recurrence: univariate analysis

\begin{tabular}{|c|c|c|c|c|c|}
\hline Variable & No. of Patients* & $10-Y r$ PFS & $p$ Value & OR & $95 \% \mathrm{Cl}$ \\
\hline Age in yrs & & & 0.353 & 1.249 & $0.781-1.999$ \\
\hline$\geq 61$ & 451 & $85.7 \%$ & & & \\
\hline$<61$ & 450 & $86.6 \%$ & & & \\
\hline Preop KPS score & & & 0.080 & 0.656 & $0.410-1.052$ \\
\hline $90-100$ & 468 & $87.3 \%$ & & & \\
\hline$\leq 80$ & 427 & $84.2 \%$ & & & \\
\hline Sex & & & 0.015 & 1.809 & $1.121-2.920$ \\
\hline Male & 263 & $84.9 \%$ & & & \\
\hline Female & 638 & $86.8 \%$ & & & \\
\hline \multicolumn{6}{|l|}{ Location } \\
\hline Supratentorial convexity & 214 & $90.2 \%$ & 0.141 & 0.627 & $0.337-1.168$ \\
\hline Parasagittal & 137 & $87.1 \%$ & 0.118 & 1.560 & $0.893-2.726$ \\
\hline Falx & 84 & $85.4 \%$ & 0.558 & 0.762 & $0.307-1.893$ \\
\hline Olfactory groove/frontobasal & 88 & $88.6 \%$ & 0.299 & 0.586 & $0.214-1.607$ \\
\hline Suprasellar & 54 & $83.3 \%$ & 0.962 & 0.975 & $0.356-2.677$ \\
\hline Lateral sphenoid & 45 & $87.4 \%$ & 0.757 & 0.833 & $0.262-2.649$ \\
\hline Medial sphenoid & 49 & $100.0 \%$ & 0.210 & 0.046 & $0-5.685$ \\
\hline Sphenoorbital & 18 & $86.7 \%$ & 0.058 & 2.657 & $0.968-7.294$ \\
\hline Cavernous sinus/temporobasal & 22 & $70.5 \%$ & 0.018 & 3.002 & $1.208-7.458$ \\
\hline Falcotentorial & 42 & $77.6 \%$ & 0.115 & 1.961 & $0.849-4.528$ \\
\hline Petrous bone & 48 & $83.9 \%$ & 0.654 & 0.768 & $0.241-2.441$ \\
\hline Petroclival & 19 & $51.0 \%$ & $<0.001$ & 6.970 & $2.998-16.207$ \\
\hline Foramen magnum/spine & 53 & $97.7 \%$ & 0.147 & 0.232 & $0.032-1.668$ \\
\hline All skull base & 348 & $83.0 \%$ & 0.450 & 1.201 & $0.746-1.933$ \\
\hline Multiplicity & & & 0.006 & 2.566 & $1.313-5.012$ \\
\hline Yes & 58 & $67.4 \%$ & & & \\
\hline No & 843 & $87.4 \%$ & & & \\
\hline \multicolumn{6}{|l|}{ Tumor size in $\mathrm{cm}$} \\
\hline$<6$ & 794 & 87.9 & \multirow{2}{*}{$<0.001$} & \multirow{2}{*}{3.685} & \multirow{2}{*}{$2.103-6.459$} \\
\hline$>6$ & 89 & $64.4 \%$ & & & \\
\hline$>3$ & 320 & $88.1 \%$ & \multirow{2}{*}{0.001} & \multirow{2}{*}{1.890} & \multirow{2}{*}{$1.286-2.777$} \\
\hline $3-6$ & 474 & $87.6 \%$ & & & \\
\hline WHO Grade (all) & & & $<0.001$ & 4.130 & $2.758-6.185$ \\
\hline I & 716 & $90.4 \%$ & & & \\
\hline II & 174 & $69.4 \%$ & & & \\
\hline III & 11 & NA & & & \\
\hline WHO 2000/2007 Grade & & & $<0.001$ & 4.096 & $2.565-6.542$ \\
\hline I & 547 & $87.2 \%$ & & & \\
\hline II & 143 & $68.3 \%$ & & & \\
\hline III & 6 & NA & & & \\
\hline \multicolumn{6}{|l|}{ MIB-1 index $(n=741)$} \\
\hline$<5 \% \dagger$ & 574 & $87.0 \%$ & \multirow{3}{*}{$<0.001$} & \multirow{3}{*}{2.626} & \multirow{3}{*}{$1.886-3.657$} \\
\hline $5-10 \%$ & 136 & $73.0 \%$ & & & \\
\hline$>10 \%$ & 31 & $60.5 \%$ & & & \\
\hline$<2 \% \dagger$ & 71 & $89.8 \%$ & \multirow{2}{*}{$<0.001$} & \multirow{2}{*}{2.325} & \multirow{2}{*}{$1.701-3.177$} \\
\hline $2-5 \% \dagger$ & 503 & $86.0 \%$ & & & \\
\hline
\end{tabular}


TABLE 4. Predictors of 10-year PFS and tumor recurrence: univariate analysis (continued)

\begin{tabular}{|c|c|c|c|c|c|}
\hline Variable & No. of Patients* & 10-Yr PFS & $p$ Value & OR & $95 \% \mathrm{Cl}$ \\
\hline \multicolumn{6}{|l|}{ Simpson Grade } \\
\hline I & 572 & $91.8 \%$ & \multirow{3}{*}{$<0.001$} & \multirow{3}{*}{2.518} & \multirow{3}{*}{$1.914-3.311$} \\
\hline II & 199 & $81.2 \%$ & & & \\
\hline III/IV & 130 & $68.5 \%$ & & & \\
\hline III‡ & 93 & $71.8 \%$ & \multirow{2}{*}{$<0.001$} & \multirow{2}{*}{2.002} & \multirow{2}{*}{$1.635-2.451$} \\
\hline IV & 37 & $65.3 \%$ & & & \\
\hline Radiosurgery/radiotherapy & & & $<0.001$ & 3.938 & $1.885-8.227$ \\
\hline Yes & 31 & $70.6 \%$ & & & \\
\hline No & 847 & $86.6 \%$ & & & \\
\hline \multicolumn{6}{|c|}{$\begin{array}{l}\text { * Not all data were available for all patients. } \\
\dagger \text { Figures are given separately for MIB-1 index. Since the survival difference between the }<2 \% \text { and } 2 \%-5 \% \text { subgroups was not statistically } \\
\text { significant, these subsets were pooled for the multivariate analysis (Fig. } 2 \text { right, Table } 5 \text { ). } \\
\ddagger \text { Figures are given separately for Simpson grade. Since the survival difference between the Simpson Grade III and IV subsets was statistically } \\
\text { not significant, both groups were pooled for the multivariate analysis (Fig. } 1 \text { lower, Table } 5 \text { ). }\end{array}$} \\
\hline
\end{tabular}

\section{Discussion}

Many meningiomas are benign tumors and appear curable through a complete resection. However, a significant number of patients will experience tumor recurrence. Overall recurrence rates as high as $26 \%$ at 10 years after surgery for a benign meningioma have been reported. ${ }^{1}$ The degree of resection has a major impact on recurrence rates. ${ }^{5,10,13}$ In 1957, Simpson et al. published a seminal paper in which meningioma resections were classified into 5 grades: complete with resection of the dural attachment (Grade I), complete with coagulation of the dural attachment (Grade II), resection of the intracranial component leaving the dural attachment and/or any extradural tumor behind (Grade III), partial resection (Grade IV), and decompression/biopsy (Grade V). ${ }^{18}$ The relationship between extent of resection and tumor recurrence forms the rationale behind aggressive surgical strategies for the management of meningiomas. However, extensive meningioma surgery may carry substantial morbidity. ${ }^{20}$

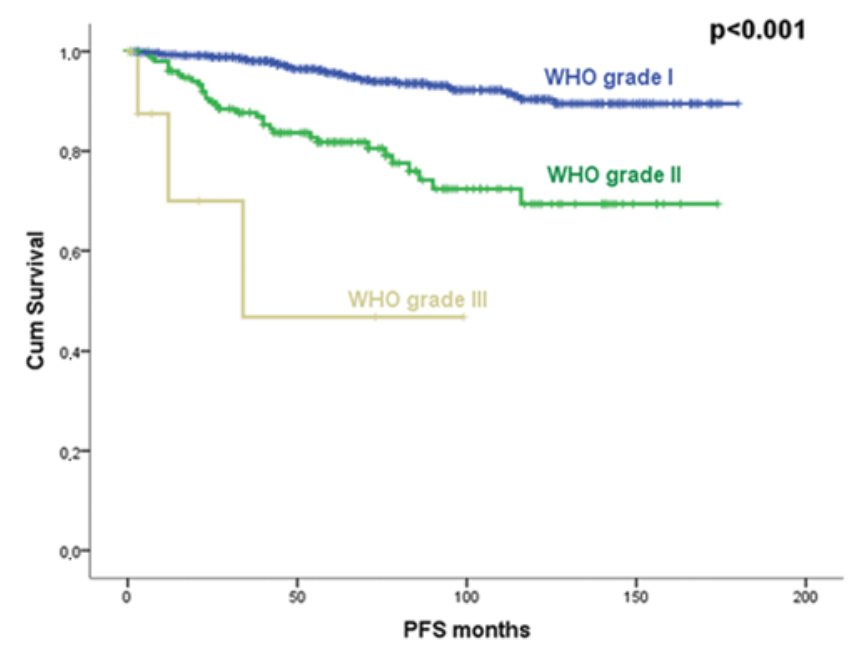

For the present paper, we have therefore analyzed our institutional experience with the impact of the degree of resection on tumor recurrence in a large series of patients ( $\mathrm{n}=901$ ) with WHO Grade I-III meningiomas versus the patients' functional outcomes. The intrinsic aggressiveness of the tumors was assessed using the proliferation marker MIB-1 in addition to the WHO grade in $82.2 \%$ of cases. Our data show that the degree of resection has a powerful impact on meningioma recurrence. The difference in terms of PFS between Simpson Grade I and II resections is a more than 2-fold increase in the recurrence rate. Tumor biology also matters. ${ }^{12}$ In addition to the WHO grade, the MIB-1 labeling index was also found to convey independent prognostic information. We obtained no evidence to suggest that more aggressive resections resulted in worse functional outcomes.

There has been a recent trend toward more conservative resection strategies for meningiomas. ${ }^{2,3,6}$ Even the clinical usefulness of the Simpson grading in general has been

FIG. 2. PFS varied significantly (left) with the histological tumor grade (WHO Grade I vs II vs III, Kaplan-Meier estimates, $p<$ 0.001 ) and (right) the MIB-1 labeling index (<2\% vs $2 \%$ to $<5 \%$ vs $5 \%-10 \%$ vs $>10 \%$, Kaplan-Meier estimates, $p<0.001$ ). The prognostic value of the abovementioned variables as independent predictors of PFS was confirmed in the multivariate analysis. Figure is available in color online only.

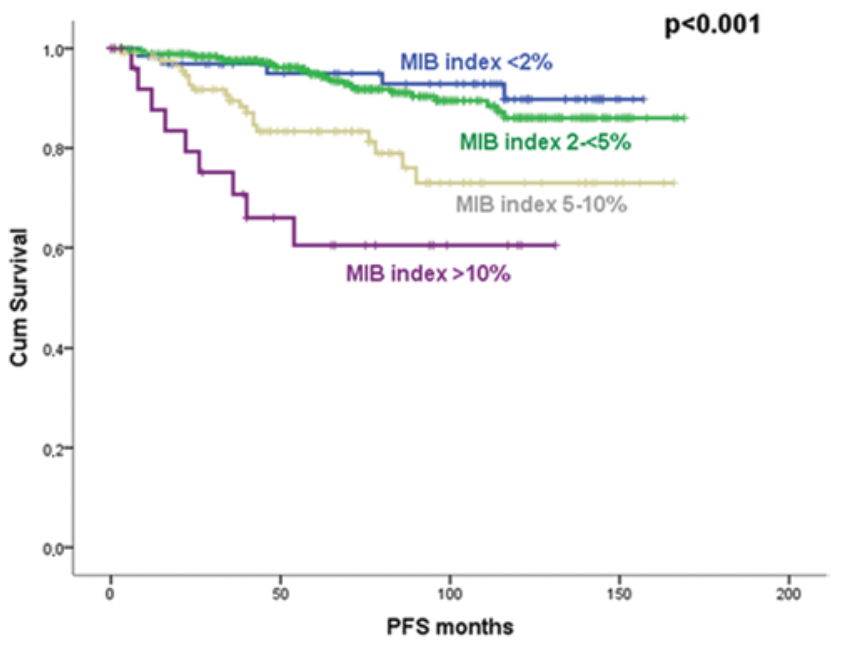


A

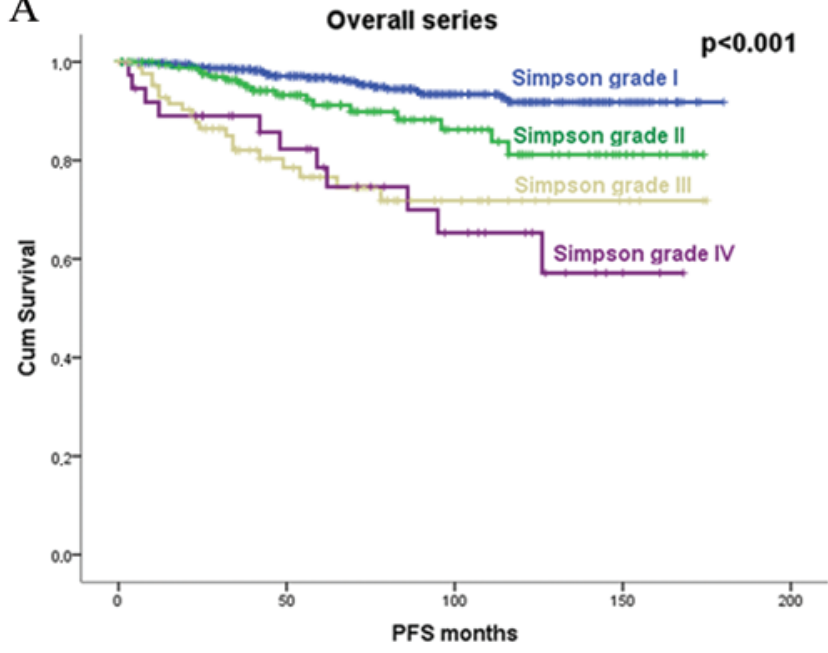

$\mathrm{C}$

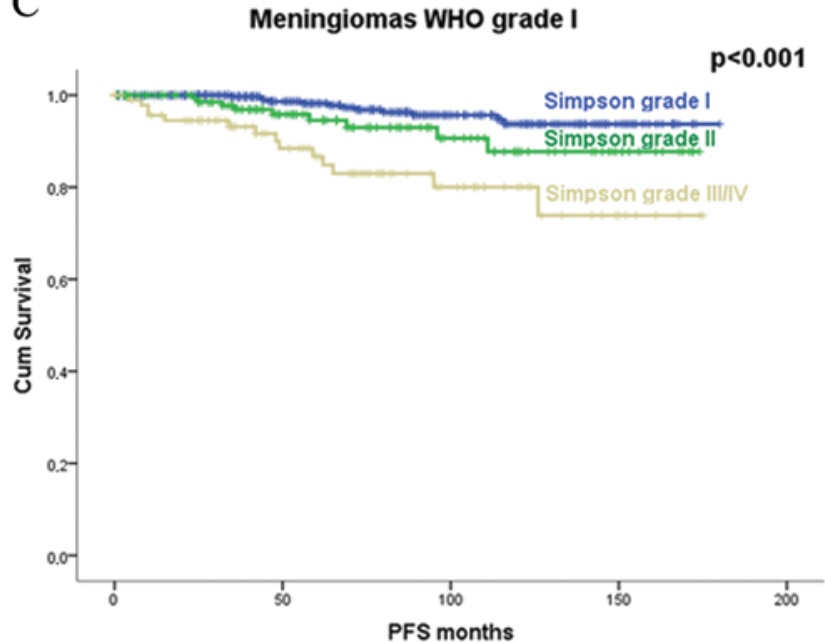

B

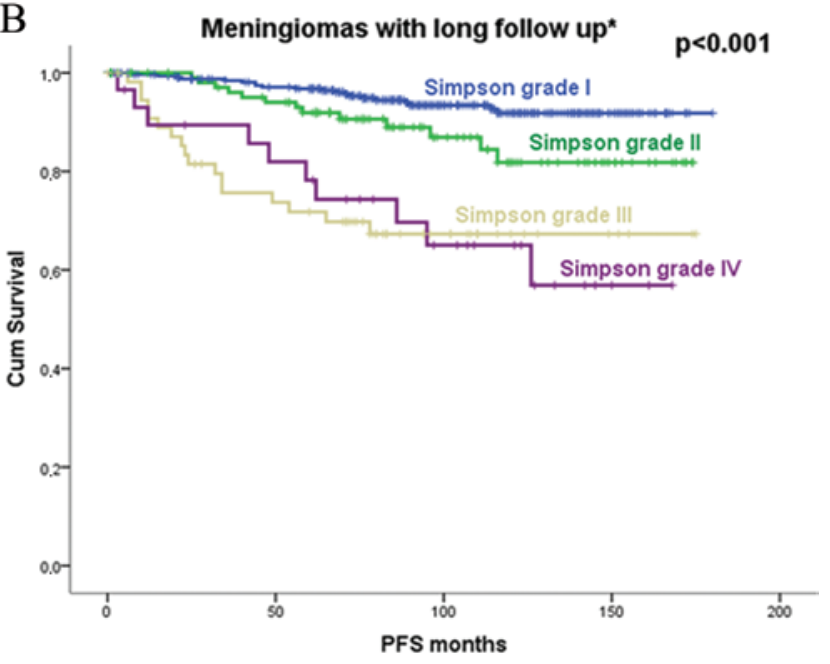

$\mathrm{D}$

Meningiomas WHO grade II

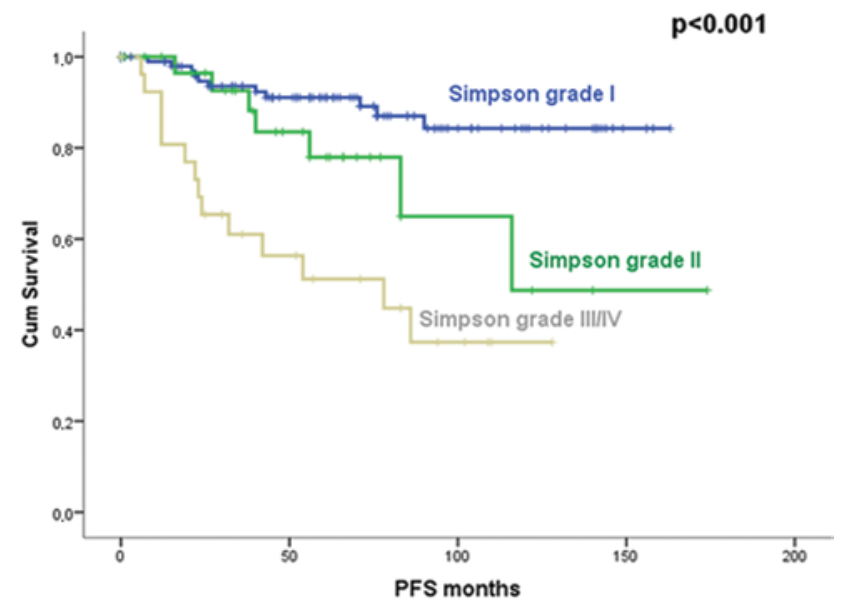

FIG. 3. The amount of resection (i.e., the Simpson grade) correlated closely with PFS in both the overall series $(n=901)(A)$ and 544 patients with a follow-up longer than 5 years or until death (B) (Kaplan-Meier estimates, both $p<0.001)$. The impact of the degree of resection increased with the WHO grade. Kaplan-Meier estimates are shown for patients with meningiomas of WHO Grade I (C) and WHO Grade II (D). For illustration purposes Simpson Grade III and IV resections were combined. Only 5 Simpson Grade IV resections were performed in patients with WHO Grade II meningiomas. Figure is available in color online only.

questioned (at least for benign meningiomas)..$^{19}$ Sughrue et al. analyzed their institutional experience with 373 patients harboring WHO Grade I meningiomas and concluded that the differences in PFS following Simpson Grade I-IV resections were not statistically significant. ${ }^{19}$ This has far-reaching consequences; instead of aiming at a tumor excision, simple tumor debulking would have to become the surgical strategy of choice given the increase in factors such as morbidity and operation time associated with more extensive resections. We were able to study a substantially larger cohort with longer follow-up. Our data show that recurrence rates indeed do vary significantly with the degree of resection while essentially providing very similar absolute figures for PFS (Fig. 3). We therefore like to think that there is a significant impact of the degree of resection on recurrence rates in benign meningiomas. However, the size of this effect can be seen in 2 ways. Some would argue that a 5\% difference in PFS at 10 years following a Simpson Grade I versus II resection does not justify aggres- sive surgery, while others would point out that this means more than doubling the risk of recurrence (Fig. 3C). A significant number of patients harbor atypical or anaplastic meningiomas ( 1 in 5 in this series) and at least atypical tumors are most often diagnosed during the postoperative neuropathological work-up rather than during surgery. Therefore, it may be more appropriate to look at combined figures for all meningiomas and not WHO Grade I tumors when planning the surgical strategy. PFS at 10 years in this series was $91.5 \%$ versus $81.2 \%$ following a Simpson Grade I versus Grade II resection (Fig. 3A). In our view this is a sizable and clinically very relevant effect.

The somewhat critical reappraisal of the routine use of the Simpson grading scheme for meningioma surgery by Sughrue et al. is not simply based on the lack of a prognostic difference between Simpson Grades I and II in their series. ${ }^{5,10,13,19}$ Sughrue et al. also pointed out that Simpson Grade IV resections are rare nowadays and will usually refer to cases with only small tumor remnants in contrast 
TABLE 5. Predictors of PFS and tumor recurrence: multivariate analysis (stepwise backward procedure)

\begin{tabular}{lrrc}
\hline \multicolumn{1}{c}{ Variable } & $p$ Value & HR & $95 \% \mathrm{Cl}$ \\
\hline Location* $^{*}<0.001$ & & \\
\hline Cavernous sinus/temporobasal & 0.009 & 3.539 & $1.372-9.130$ \\
\hline Petroclival & $<0.001$ & 8.363 & $3.341-20.935$ \\
\hline Multiplicity: yes & 0.010 & 2.594 & $1.262-5.332$ \\
\hline Tumor size $>6 \mathrm{~cm}$ & 0.003 & 2.658 & $1.402-5.039$ \\
\hline WHO Grade & 0.011 & & \\
\hline WHO Grade II & 0.003 & 2.923 & $1.436-5.949$ \\
\hline WHO Grade III & 0.074 & 3.927 & $0.877-17.582$ \\
\hline MIB-1 index & 0.017 & & \\
\hline MIB-1 index 5-10\% & 0.485 & 1.310 & $0.614-2.794$ \\
\hline MIB-1 index $>10 \%$ & 0.006 & 3.784 & $1.462-9.797$ \\
\hline Simpson Grade & $<0.001$ & & \\
\hline Simpson Grade II & 0.006 & 2.665 & $1.333-5.328$ \\
\hline Simpson Grade III/IV & $<0.001$ & 6.264 & $3.331-11.781$ \\
\hline * Locaton: Cavernous & &
\end{tabular}

* Location: Cavernous sinus/temporobasal versus petroclival versus all others.

to the times of Simpson. Indeed, the prognostic difference between Simpson Grade III and IV resections was far from convincing in the present series, and it may well be clinically sufficient to simply distinguish between Grades I, II, and all others. The Simpson grading scheme when applied to different tumor characteristics, such as location, growth pattern, and size, may not be entirely consistent. A Simpson Grade I resection of a convexity meningioma probably refers to a much more radical tumor resection than a Simpson Grade I resection in the skull base. We would agree with Sughrue and coworkers that more location-specific classification schemes may prove useful.

The most convincing argument in favor of conservative meningioma surgery appears to be the presumed association between substantially increased surgical morbidity and extensive operations. ${ }^{14,20}$ Our data provide no statistical support for this concept. The degree of resection even correlated inversely with the patients' functional outcome in the univariate analysis; more extensive surgery was associated with a better rather than a lower postoperative KPS score. The degree of resection was not an independent predictor of the patients' functional outcome in the multivariate analysis. We routinely resected the area of meningeal enhancement in convexity tumors. Surgery for parasagittal meningiomas often involved resection of the lateral edge of the sagittal sinus. The sinus itself was rarely entered and only occasionally reconstructed. We resected rather than coagulated the dural attachment in frontobasal meningiomas. Surgery for sphenoid meningiomas often involved extensive bone removal and dural reconstruction. We did not routinely enter the cavernous sinus. We are aware of the methodological restrictions imposed by a retrospective study that does not compare different operative strategies but rather looks for correlations between the extent of resection and outcomes in a single-center/singlepolicy cohort.

Our data also illustrate that meningioma recurrence is a function of tumor biology. The 3-tiered WHO classifica- tion scheme appears to distinguish well between patient groups characterized by low, intermediate, and high recurrence rates. Of note, this was true for tumors diagnosed before and after 2000, i.e., before and after the WHO 2000 revision of the meningioma grading scheme. We will readily acknowledge that one of the shortcomings of this study is the lack of a neuropathological review. Interestingly, we found that the MIB-1 proliferation index provides additional prognostic information independent of the WHO grade. The intrinsic aggressiveness of a meningioma is only incompletely assessed by the WHO classification. Future revisions of the WHO classification may have to again consider incorporating the MIB-1 index as a grading parameter. On the other hand, it may well be worthwhile to look beyond the MIB-1 index for other prognostic biomarkers. Deletions and loss of heterozygosity of markers from $1 \mathrm{p}, 10$, and $14 \mathrm{q}^{7,9,17}$ have been investigated as potential predictors of tumor recurrence. Karyopherin expression has been recently correlated with the WHO grade and tumor progression in meningiomas and can be easily analyzed using immunohistochemistry. ${ }^{4}$

We will readily acknowledge several limitations of the present investigation. This is a retrospective study. We report postoperative functional outcomes, but we do not have information with respect to the clinical status of the patients later on. Because of the rather chronic character of the disease, it would be clearly desirable to have more complete follow-up information and longer follow-up duration. Most importantly, the principal oncological outcome parameter used in this analysis was tumor recurrence. Tumor recurrence does not always equal new symptoms and additional therapy, and its impact on a patient's quality of life will vary. Recurrent or progressive disease can often be controlled by a second surgery or even radiosurgery (or other forms of focused radiotherapy).

\section{Conclusions}

Despite all these limitations, our data nevertheless show that the traditional view of a radical tumor resection including its dural attachments and any extradural extensions as the best treatment for many meningiomas still has its merits. Aggressive surgery is not necessarily associated with worse functional outcomes. It will translate into superior PFS, most notably in nonbenign tumors, which are often diagnosed only after surgery and which are not as a rare as often thought. Neurosurgeons should not be deterred from pursuing aggressive meningioma resections, whenever such a strategy appears reasonably safe.

\section{References}

1. Abry E, Thomassen IO, Salvesen OO, Torp SH: The significance of Ki-67/MIB-1 labeling index in human meningiomas: a literature study. Pathol Res Pract 206:810-815, 2010

2. Combs SE, Edler L, Burkholder I, Rieken S, Habermehl D, Jäkel O, et al: Treatment of patients with atypical meningiomas Simpson grade 4 and 5 with a carbon ion boost in combination with postoperative photon radiotherapy: the MARCIE trial. BMC Cancer 10:615, 2010

3. Combs SE, Ganswindt U, Foote RL, Kondziolka D, Tonn JC: State-of-the-art treatment alternatives for base of skull meningiomas: complementing and controversial indications for neurosurgery, stereotactic and robotic based radiosurgery 
or modern fractionated radiation techniques. Radiat Oncol 7:226, 2012

4. Gousias K, Niehusmann P, Gielen GH, Simon M: Karyopherin a 2 and chromosome region maintenance protein 1 expression in meningiomas: novel biomarkers for recurrence and malignant progression. J Neurooncol 118:289-296, 2014

5. Jääskeläinen J: Seemingly complete removal of histologically benign intracranial meningioma: late recurrence rate and factors predicting recurrence in 657 patients. A multivariate analysis. Surg Neurol 26:461-469, 1986

6. Kaul D, Budach V, Wurm R, Gruen A, Graaf L, Habbel P, et al: Linac-based stereotactic radiotherapy and radiosurgery in patients with meningioma. Radiat Oncol 9:78, 2014

7. Ketter R, Henn W, Niedermayer I, Steilen-Gimbel H, König $\mathrm{J}$, Zang KD, et al: Predictive value of progression-associated chromosomal aberrations for the prognosis of meningiomas: a retrospective study of 198 cases. J Neurosurg 95:601-607, 2001

8. Louis DN, Ohgaki H, Wiestler OD, Cavenee WK, Burger PC, Jouvet A, et al: The 2007 WHO classification of tumours of the central nervous system. Acta Neuropathol 114:97-109, 2007

9. Maillo A, Orfao A, Sayagues JM, Diaz P, Gómez-Moreta JA, Caballero M, et al: New classification scheme for the prognostic stratification of meningioma on the basis of chromosome 14 abnormalities, patient age, and tumor histopathology. J Clin Oncol 21:3285-3295, 2003

10. McGovern SL, Aldape KD, Munsell MF, Mahajan A, DeMonte F, Woo SY: A comparison of World Health Organization tumor grades at recurrence in patients with non-skull base and skull base meningiomas. J Neurosurg 112:925933, 2010

11. Ostrom QT, Gittleman H, Farah P, Ondracek A, Chen Y, Wolinsky Y, et al: CBTRUS statistical report: Primary brain and central nervous system tumors diagnosed in the United States in 2006-2010. Neuro Oncol 15 (Suppl 2):ii1-ii56, 2013

12. Oya S, Kawai K, Nakatomi H, Saito N: Significance of Simpson grading system in modern meningioma surgery: integration of the grade with MIB-1 labeling index as a key to predict the recurrence of WHO Grade I meningiomas. $\mathbf{J}$ Neurosurg 117:121-128, 2012

13. Palma L, Celli P, Franco C, Cervoni L, Cantore G: Longterm prognosis for atypical and malignant meningiomas: a study of 71 surgical cases. J Neurosurg 86:793-800, 1997

14. Seifert V: Clinical management of petroclival meningiomas and the eternal quest for preservation of quality of life: personal experiences over a period of 20 years. Acta Neurochir (Wien) 152:1099-1116, 2010
15. Simon M, Boström J, Koch P, Schramm J: Interinstitutional variance of postoperative radiotherapy and follow up for meningiomas in Germany: impact of changes of the WHO classification. J Neurol Neurosurg Psychiatry 77:767-773, 2006

16. Simon M, Park TW, Leuenroth S, Hans VH, Löning T, Schramm J: Telomerase activity and expression of the telomerase catalytic subunit, hTERT, in meningioma progression. J Neurosurg 92:832-840, 2000

17. Simon M, von Deimling A, Larson JJ, Wellenreuther R, Kaskel P, Waha A, et al: Allelic losses on chromosomes 14, 10 , and 1 in atypical and malignant meningiomas: a genetic model of meningioma progression. Cancer Res 55:46964701, 1995

18. Simpson D: The recurrence of intracranial meningiomas after surgical treatment. J Neurol Neurosurg Psychiatry 20:22-39, 1957

19. Sughrue ME, Kane AJ, Shangari G, Rutkowski MJ, McDermott MW, Berger MS, et al: The relevance of Simpson Grade I and II resection in modern neurosurgical treatment of World Health Organization Grade I meningiomas. J Neurosurg 113:1029-1035, 2010

20. Zentner J, Meyer B, Vieweg U, Herberhold C, Schramm J: Petroclival meningiomas: is radical resection always the best option? J Neurol Neurosurg Psychiatry 62:341-345, 1997

\section{Disclosures}

The authors report no conflict of interest concerning the materials or methods used in this study or the findings specified in this paper.

\section{Author Contributions}

Conception and design: all authors. Acquisition of data: Gousias. Analysis and interpretation of data: Gousias, Simon. Drafting the article: all authors. Critically revising the article: all authors. Approved the final version of the manuscript on behalf of all authors: Gousias. Statistical analysis: Gousias, Simon. Study supervision: Gousias.

\section{Correspondence}

Konstantinos Gousias, Department of Neurosurgery and Neurotraumatology, University Hospital of Bochum Bergmannsheil, Bürkle de la Camp Platz 1, 44789, Bochum, Germany.email: kostasgousias@yahoo.com. 